\title{
A Cognitive Method for Musicology Based Melody Transcription
}

\author{
Jiayin Sun* \\ Harbin Institute of Technology, Harbin 150001, China \\ Hongyan Wang \\ Heilongjiang University, Harbin 150001, China \\ E-mail:2010013@hlju.edu.cn
}

Received 4 July 2015

Accepted 8 October 2015

\begin{abstract}
This paper describes a method for transcribing the main structure of polyphonic music audio automatically by analyzing musical tonality related musicological information. Music transcription is a difficult topic in Music Information Retrieval (MIR) which contains many tasks to recognize all the elements of music. Among all these musical factors, tonality is a basic but elusive one, and plays as the core in organizing music structure. So by making an investigation into cognition and musicology, we propose an approach to transcribe major component of polyphonic music from the point of view of tonality. Unlike previous studies, our method focuses on music cognition theory instead of signal processing and pattern classification. Basing on our former work on key finding and chord recognition, we introduce a new cognitive feature called auditory saliency (AS), which contains both statistically modeled information about human's acoustic attention and psychology measured data on human's musical perception, to recognize the main part of the music - melody stream. Constant Q transform which is a more "musical" STFT (Short-Time Fourier Transform), and a temporal ANN (Artificial Neuro Network) are also used in our framework. There are also a few musicology and signal processing based techniques designed to improve our method such as melody structural vertical regulating and onset detection. Our proposed method has been tested objectively (F-measure of pitch detection is 0.76 with a perfect musicological AS information) and subjectively (above $90 \%$ of transcribed segments are accepted by professional reviewers), and shows some inspiring results.
\end{abstract}

Keywords: Auditory saliency, tonal analysis, music cognition, melody stream transcription.

\section{Introduction}

Music transcription refers to extraction of a human readable and interpretable description from a recording of a music performance. ${ }^{1}$ Traditionally speaking, it can be viewed as a technique which is able to convert music audio into score based representation of music work. It needs solutions to detect music elements from every aspect, such as key finding, melody extraction, chord recognition, and so on. Music transcription is a complex topic, even human with music background are incapable of promising their accuracy when doing the job. And the sub-tasks of music transcription have been studied for years in MIR and related fields. ${ }^{2}$ Due to the situation, transcribing the main structure of music is an acceptable alternative in the primary stage of music transcription study.

\subsection{Related work}

In traditional studies of music transcription (including sub-tasks), pattern recognition method is

\footnotetext{
"Corresponding author, sunjiayin@hit.edu.cn
} 
the focus. $\mathrm{Zhu}^{3}$ presents a method for pitch profile extraction in key finding, which deals with pitch mistuning and noisy percussive sounds; Gomez ${ }^{4}$ proposes harmonic pitch class profile to detect key. Goto $^{5}$ proposes a pitch estimation method: the influential method calculates the most predominant F0 by regarding input frequency components as a weighted mixture of all possible F0s (fundamental frequency), then simultaneously estimates their weights by MAP (Maximum A Posteriori) algorithm. Scholars ${ }^{6,7}$ both study the multi-pitch estimation problem: the former proposes a method consists of a computational model of the human auditory periphery, followed by a periodicity analysis mechanism where fundamental frequencies are iteratively detected and canceled from the mixture signal; while the latter addresses the issue of overlapping overtones by modeling the spectral envelope of the overtones of each note with a smooth autoregressive mode. Lee ${ }^{8}$ uses a database of symbolic musical scores to obtain chord ground truth and acoustic waveforms. Furthermore, there are also achievements in studying rhythm detection topic. ${ }^{2}$ For music transcription, a representative one is Cemgil's work ${ }^{1}$, which has considered musical attributes mentioned above. With respect to melody focused work, $\mathrm{Jo}^{9}$ proposes a harmonic summation to pick the multi-pitch, and rule based method to track specific voice part. Yeh ${ }^{10}$ uses a harmonic-percussive sound separation (HPSS) based method to perform melody-accompaniment separation and MultiResolution Fast Fourier Transform (MRFFT) to process the music signal. Arora ${ }^{11}$ applies STFT with $\log$ spectrum, and threshold to detect salient part. Salamon $^{12,13}$ also does great jobs in multi-pitch selection and tracking.

On the other hand, music cognition may offer a different solution to music transcription. Music cognition refers to a loose affiliation among several established disciplines, including psychology, musicology, and neuroscience. Unlike traditional computing ideas, music cognition is based on psychological experiments and statistical analysis. Psychologists have investigated in this field by observing the human's reaction after hearing music, and seeking for interpretation in musicology and neuroscience. Krumhansl ${ }^{14}$ proposes a famous key profile, which is based on psychological experiments designed with "probe tone", and applies the profile in key finding model. Chew ${ }^{15}$ proposes a "spiral array" to represent the quantitative relations among pitches, intervals, chords, and keys. Terhardt ${ }^{16}$ proposed a model that predicts root for complex tonal Stimuli by analyzing the structure of harmonic. Temperley ${ }^{17}$ proposed a distributional key finding model based on probabilistic reasoning.

\subsection{Contribution of this paper}

However, there are key problems which are still unsolved in music computing and related areas. Firstly, from the cognitive point of view, we have no idea how human perceives music, and how brain processes musical and audio information in neuro level. Secondly, computationally speaking, since even human with professional background aren't able to precisely locate the salient pitch or major elements in polyphonic music, we have to deal with complicated harmonic structure. Finally, no predominant musicological model has been established, so we can't take full advantage of musicology. On the other hand, psychological experiments ${ }^{14,18,19}$ show that selective attention does exist in human's perception of music, and it is context dependent. So this paper proposes a "musical" method to transcribe salient part of music (melody stream), with inspiration of cognition and musicology. Auditory saliency, a cognitive feature contains information about both statistical measurement and musicological cause of selective attention in music, is proposed to detect the melody stream structure from harmonic sequence. A temporal ANN is introduced to simulate human tonality perception mechanism. In order to detect precise tones, Our previous researches on key finding ${ }^{20}$ and chord recognition ${ }^{21}$ are presented. Pitch tuning and onset detection based on $\mathrm{CQT}^{22}$ are used to make the system robust. Unlike our last $\mathrm{study}^{23}$, which simply proposed the basic idea of auditory saliency detection method, this paper introduces more musical knowledge into our method, by adding a melody structural vertical regulating post-processing stage based on musicological rules.

The remainder of the paper is structured as follows: In section 2, a cognitive feature AS, is introduced. Section 3 discusses the framework of our melody stream transcription system and the post-processing method. 
Experiment results are included in section 4. Section 5 concludes this paper.

\section{Auditory Saliency and Music Cognition}

\subsection{Auditory saliency}

When hearing a mixed sound, human pays attention mainly on some parts of the sound that neural system considers to be "important". The phenomena are called selective attention. Music can be regarded as a temporal sequence of mixed sounds, so selective attention should explain music perception in some aspects, and psychological experiments ${ }^{14}, 18,19$ have proved it. Furthermore, selective attention based applications have been made in computer vision. ${ }^{24,25}$ This paper explains it simply as following: acoustical selective attention is related to organization of the mixed sound, as well as relations between components of the mixture, and changes of each component; furthermore, context contributes to selective attention in music. Components "selected" by attention are salient pitches in sound mixtures, and other components function so inconsiderably that listeners choose to "forget" them. So transcribing the salient parts of music audio only is reasonable when current MIR related fields still develop insufficiently.

According to our theory on musical selective attention, 2 factors should be considered to select the salient parts in the mixture (chord) at a given time-auditory factor that makes the parts prominent, and musical factor that reinforces the saliency. Thus, we propose a cognitive feature, auditory saliency, to measure the extent of the selective attention.

AS is a kind of special mapping from objective audible set to subjective result set of perception:

$$
f_{A S}: O \rightarrow C
$$

Here $O$ is an event set (order is not necessary) in the real world, commonly, it is a set of temporal sequences of audible events. $C$ is an ordered set of perception results of $O$ in human's cognitive space, and its order is followed by neural system to make response after processing the captured information from $O$. The auditory saliency function $f_{A S}$ is a sort mapping by simulating human's behavior of perception, and it transforms each audible event in $O$ into $C$ in some order according to its "importance" (here, AS).

With our definition of AS, selective attention can be explained as following: Given an $O$ (sound mixture), establish $f_{A S}$ with measurement of AS, then map $O$ into $C$ following the order based on AS. Here we don't know exactly how $f_{A S}$ functions, but if we find a proper AS, then the order of $C$ is determined, and the salient parts of sound is recognized.

\subsection{Acoustical measurement of $A S$}

For a given sound mixture set (let's say $O$ ), there are evidences $^{24,25,26}$ that more attractive events are those appear distinguished from "surroundings". In music, given a sound mixture and a pitch that is a component of the mixture, we regard this kind of distinctness as differences between the pitch and other pitches at the same time (structured difference), plus differences between the pitch and pitches adjacent in time (temporal difference). In this way, we can define AS.

Here, $O$ is actually a temporal sequence, in order to calculate AS, we introduce a temporal Bayesian inference, at time $t+1$ :

$$
P\left(T_{t+1} \mid O_{t+1}\right)=\frac{P\left(O_{t+1} \mid T_{t+1}\right)}{P\left(O_{t+1}\right)} \cdot P\left(T_{t+1}\right)
$$

From perspective of cognition, the prior distribution of background information space at time $t, T_{t}$ is based on the subjective perception of human, and changes by being updated to posterior distribution with the new arrival data $O_{t}$. And this posterior distribution at time $t$ is actually the prior distribution at time $t+1$, which means:

$$
P\left(T_{t+1}\right)=P\left(T_{t} \mid O_{t}\right)
$$

Combine the above formula, we have:

$$
P\left(T_{t+1} \mid O_{t+1}\right)=\frac{P\left(O_{t+1} \mid T_{t+1}\right)}{P\left(O_{t+1}\right)} \cdot P\left(T_{t} \mid O_{t}\right)
$$

Here $P\left(T_{t} \mid O_{t}\right)$ is the posterior distribution of our temporal sequence $O$ at time $t$ over background information space $T_{t}$. When new observed data $O_{t+1}$ comes, we take $P\left(T_{t} \mid O_{t}\right)$ as the prior probability distribution over $T_{t+1}$. Then $P\left(T_{t+1} \mid O_{t+1}\right)$ is calculated via Bayes theorem. Thus, the posterior distribution $P\left(T_{t+1} \mid O_{t+1}\right)$ is related only to the situation at time $t$. Furthermore, according to the conjugate prior $^{27}$, Gaussian family is self-conjugate with respect to Gaussian likelihood function. Thus, if distribution of the likelihood function $P\left(O_{t+1} \mid T_{t+1}\right)$ is a normal distribution with a known variance, then a Gaussian prior distribution $P\left(T_{t} \mid O_{t}\right)$ will lead to a Gaussian posterior distribution $P\left(T_{t+1} \mid O_{t+1}\right)$.

To calculate proper measurement of AS, implementing CQT signal transformation in a frequency range of $110.0 \mathrm{~Hz}-3951.1 \mathrm{~Hz}$, which means A2-B7 in tone and contains 63 semitones. $x_{O}[t, k]$ denotes the new coming 
observed data of bin $k$ at time $t$ on the spectrum, for each channel at time $t$ that is 100 cents ( 1 semitone) frequency range wide centers at the tone of chromatic scale (each channel represents a tone), suppose the data in the channel is Gaussian distributed. The likelihood function $P\left(O_{t} T_{t}\right)$ is new data distribution over subjective cognition space:

$$
P\left(O_{t} \mid T_{t}\right)=P\left(x_{o}[t, k]\right), x_{o}[t, k] \propto G\left(\bar{\mu}_{i}(t), \sigma^{2}\right) i=1,2, \ldots, 61
$$

Here $k \in[-50+100 i, 50+100 i]$, the mean $\overline{\mu_{l}}(t)$ is unknown, and standard deviation $\sigma$ is known. In the ideal case, the mean should be at the channel center; however, it has to be estimated from the observed data at time $t$ and possibly deviates from channel center. Furthermore, according to our experiment $t^{20}$, about $68 \%$ of data is distributed within 15 cents from the center. Thus, with the characteristic of normal distribution, $\sigma$ is set to be 15 . Since likelihood function $P\left(O_{t} \mid T_{t}\right)$ is normally distributed with known standard deviation, a normally distributed $P\left(T_{t} \mid O_{t}\right)$ leads to a Gaussian $P\left(T_{t+1} \mid O_{t+1}\right)$.

For $x[t, k]$ in each channel at time $t$ :

$$
P\left(T_{t} \mid O_{t}\right)=P(x[t, k]), x[t, k] \propto G\left(\mu_{i}(t), \sigma_{i}(t)^{2}\right)
$$

Thus, at time $t+1$,

$$
P\left(T_{t+1} \mid O_{t+1}\right)=P(x[t+1, k]), x[t+1, k] \propto G\left(\mu_{i}(t+1), \sigma_{i}(t+1)^{2}\right)
$$

According to conjugate prior, parameters of Gaussian at $t+1$ can be calculated with parameters at $t$ and those of observed distribution at $t+1$ :

$$
\begin{gathered}
\mu_{i}(t+1)=\left(\frac{\mu_{i}(t)}{\sigma_{i}(t)^{2}}+\frac{\bar{\mu}_{i}(t)}{h^{2} \sigma^{2}}\right) /\left(\frac{1}{\sigma_{i}(t)^{2}}+\frac{1}{h^{2} \sigma^{2}}\right) \\
\sigma_{i}(t+1)^{2}=\left(\frac{1}{\sigma_{i}(t)^{2}}+\frac{1}{h^{2} \sigma^{2}}\right)^{-1}
\end{gathered}
$$

Here, denoising factor $h(h \in[1,6])$ is selected by the following experiments: randomly and repeatedly consider channels from the most salient ones (e. g. the root channel) to the least salient ones (e. g. neither salient nor with salient neighbors). In each situation, 2 most obvious local maximums measured in a 10-bin width are selected, then the mean of 10 bins of local maximum is compared with the mean of the channel. In this way, we obtain the relations of means between local maximum and channel in different salient levels. And we use $h$ to describe the level of channel saliency. For simplicity, we divide channel saliency into 6 levels: $h=1$ means the observed data is effective and distributed closely around the center of the channel (most salient); and $h=6$ makes observed data least effective and dispersedly distributed (when $h=6.67, h \sigma>100$, deviation of observed data will exceed the channel, least salient).

For channel $i$ at time $t$, let $\overline{x_{l}}$ be the mean of the channel:

$$
\overline{x_{i}}=\sum_{k=0}^{99} x[t, 100 i+k-50]
$$

And $\overline{\overline{x_{l}}}$ denotes the mean of arbitrary 10 continuous bins:

$$
\overline{\overline{x_{i}}}=\sum_{k=0}^{9} x[t, 100 i+k+m], m \in(-50,40) \text { (11) }
$$

Here $\max \left(\overline{\overline{x_{l}}}\right)$ denote the largest possible value of $\overline{\overline{x_{l}}}$, then $h$ is determined as the Table 1 .

Table 1. Possible values for $h$

\begin{tabular}{cc}
\hline Constraints for selection of $h$ & Selection of $h$ \\
\hline No $\overline{\overline{x_{i}}}$ makes $\overline{\overline{x_{i}}}>1.10 \overline{x_{i}}$ & 5 \\
$1.10 \overline{x_{i}}<\max \left(\overline{\overline{x_{i}}}\right) \leq 1.17 \overline{x_{i}}$ & 4 \\
$1.17 \overline{x_{i}}<\max \left(\overline{\overline{x_{i}}}\right) \leq 1.23 \overline{x_{i}}$ & 3 \\
$1.23 \overline{x_{i}}<\max \left(\overline{\overline{x_{i}}}\right) \leq 1.28 \overline{x_{i}}$ & 2 \\
$1.28 \overline{x_{i}}<\max \left(\overline{\overline{x_{i}}}\right) \leq 1.32 \overline{x_{i}}$ & 1 \\
$\max \left(\overline{\overline{x_{i}}}\right)>1.32 \overline{x_{i}}$ &
\end{tabular}

We have calculated every channel's distribution at each time by taking its distribution at last time stamp as prior distribution. If the music sound at a time contains the tone the channel represents, distribution of the channel at this time must be different. Here we introduce the KullbackLiebler distance ${ }^{28}$ to capture the differences between distributions from 2 aspects and measure it as the cognitive feature AS.

As our definition above, we here calculate 2 kinds of AS-local AS (structured difference) and temporal AS (temporal difference). Local AS, which is denoted by $A S_{L}$, measures the acoustical difference of a given channel from that of its neighboring channels at a given time.

$$
\begin{aligned}
A S_{L}(t, i) & =\frac{D_{K L}\left(G\left(\mu_{i}(t), \sigma_{i}(t)^{2}\right), G\left(\mu_{i-1}(t), \sigma_{i-1}(t)^{2}\right)\right)}{2} \\
& +\frac{D_{K L}\left(G\left(\mu_{i}(t), \sigma_{i}(t)^{2}\right), G\left(\mu_{i+1}(t), \sigma_{i+1}(t)^{2}\right)\right)}{2}
\end{aligned}
$$

Here:

$$
\begin{aligned}
& D_{K L}\left(G\left(\mu_{i}(t), \sigma_{i}(t)^{2}\right), G\left(\mu_{i-1}(t), \sigma_{i-1}(t)^{2}\right)\right) \\
& =\int_{-\infty}^{+\infty} \frac{e^{-\frac{\left(x-\mu_{i}(t)\right)^{2}}{2 \sigma_{i}(t)^{2}}}}{\sqrt{2 \pi} \sigma_{i}(t)} \cdot \log \left(\frac{\sigma_{i-1}(t)}{\sigma_{i}(t)} \cdot e^{\frac{\left(x-\mu_{i-1}(t)\right)^{2}}{2 \sigma_{i-1}(t)^{2}}-\frac{\left(x-\mu_{i}(t)\right)^{2}}{2 \sigma_{i}(t)^{2}}}\right) \cdot d x
\end{aligned}
$$


a)

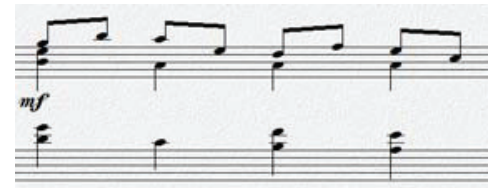

b)

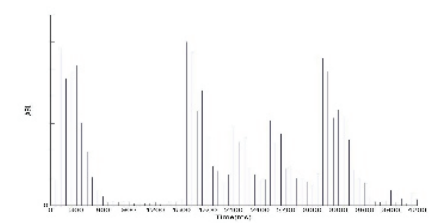

c)

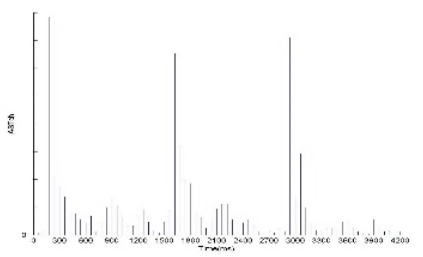

d)

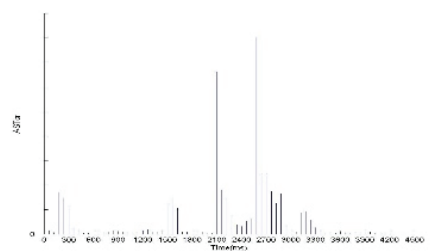

e)

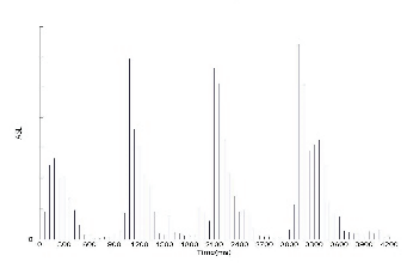

f)

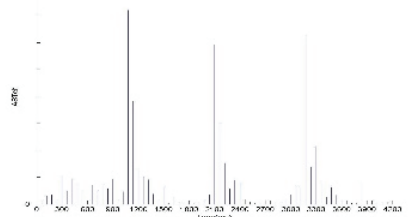

g)

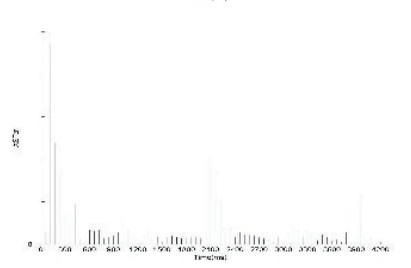

Fig. 1. An example of AAS results for 2 channels in the first bar of a new age song: a) First bar of the new age piece. b) $A S_{L}$ pattern of the $32^{\text {nd }}$ subband (note E5). c) $A S_{T-c h}$ pattern of the $32^{\text {nd }}$ subband(note E5). d) $A S_{T-c r}$ pattern of the $32^{\text {nd }}$ subband (note E5). e) $A S_{L}$ pattern of the $25^{\text {th }}$ subband (note A4). f) $A S_{T-}$ ch pattern of the $25^{\text {th }}$ subband (note A4). g) $A S_{T-c r}$ pattern of the $25^{\text {th }}$ subband (note A4).
Note that K-L distance is not symmetric, and we always regard the distribution of the target channel as the former item in calculation.

Temporal AS, $A S_{T}$, describes acoustical change of a channel as time passes. To capture the temporal difference exactly, we propose 2 measurements-- $A S_{T-c h}$ (difference between contiguous time stamps in a channel), and $A S_{T-c r}$ (dynamic contrast between target channel and its neighbors concomitant with change of target channel).

$$
\begin{gathered}
A S_{T-c h}(t, i)=D_{K L}\left(G\left(\mu_{i}(t), \sigma_{i}(t)^{2}\right), G\left(\mu_{i}(t-1), \sigma_{i}(t-1)^{2}\right)\right) \\
A S_{T-c r}(t, i)=\frac{A S_{T-c h}(t, i+1)+A S_{T-c h}(t, i-1)}{2}
\end{gathered}
$$

Thus, we get the acoustical measurement of AS, a 3-D vector, AAS:

$$
\text { Acoustical } A S=\left(\begin{array}{c}
A S_{L} \\
A S_{T-c h} \\
A S_{T-c r}
\end{array}\right)
$$

Due to the implementation of AAS calculation, $A S_{T-c h}$ is very sensitive to onset of note represented by its channel; although $A S_{L}$ is less efficient for onset, it remains prominent as note of its channel sounds; and $A S_{T-c r}$ successfully describes the contrast between target channel and its neighbors, but one potential problem is that if there are many components in mixed music sound (e. g. different instruments), $A S_{T-c r}$ may lose its local maximum. Fig. 1 is an example of AAS results for 2 channels in the first bar of a new age song.

\subsection{Acoustical measurement of $A S$}

Tonality is one of the main conceptual categories in western musicology. In the broadest possible sense, however, it refers to systematic arrangements of pitch phenomena and relations between them. ${ }^{18,29}$ Tonality can be abstracted as a separate theoretical system in which most of musical elements are well organized, and especially it has shown a powerful explanation to human's cognition of music in psychological experiments. $^{29}$ So we propose some musical measurements of perception based on tonality.

When listening to a note (chord) in music, neural system doesn't only process it separately, but also put it in the context, which means human's perception of music elements is considerably affected by music context, according to researches. ${ }^{14,15,17,18,19}$ So when we consider 
if a channel at a time is salient, it is necessary to examine the channel's musicological saliency in context.

Especially, human's judgment of a tone is heavily influenced by what she has heard just now. ${ }^{18}$ Hence, difference between the channel at a given time and key of the context before this time is considered, which is denoted by $A S_{N K} . A S_{N K}$ is measured based on the famous experiment "probe-tone". ${ }^{14}$ In the experiment, participants heard, at various times, major scales, minor scales, tonic triads, or prototypical chord progressions ending on the tonic. The listeners responded to each probe by rating, on a scale from one to seven, how well the tone fit with the preceding context. Then profile for each key is made with the results of rating averaged for probe tones following the contexts of triads and chord progressions (K-K key profile, for instance, see Fig. 2). In both major and minor contexts, the tonic pitch received the highest rating, the tonic triad's two other tones received the next highest ratings, and the remaining tones of the diatonic scale all received ratings higher than those registered for any nondiatonic tone. Clearly these ratings echo the relative degrees of stability traditionally ascribed by music theorists to each semitone in the context of a major or minor key. Furthermore, with many replications, ${ }^{18}$ the profiles have proven to be effective to measure difference between keys.

Here we use $K(i, k)$ to denote rating of tone $i$ with respect to key $k$ in the K-K profile. Thus, giving channel $i$ at time $t$, to measure the distance between a channel and its previous context, we define $A S_{N K}$ in (17).

$$
\begin{aligned}
& A S_{N K}(t, i)=K((i+10) \bmod 12, k(t)) \\
& i=1,2, \ldots, 61
\end{aligned}
$$

Here $k(t)$ is music key at time $t$ determined by our proposed system (we use ANN to classify keys on CQT spectrum, labeling the key at every time stamp of input music segment) in our previous study ${ }^{20}$, and is treated as given background information. And $(i+10) \bmod 12$ represents the relative tone position of channel $i$ in an octave.

After checking if a channel fits with its previous context, we should focus on the cognitive stability of a channel with respect to its current tonal structure. That is, the degree of cognitive consonance human perceives when she hears the new coming sound mixture (current chord) which contains the target channel. Generally, saliency pitch candidates are those that make current tonal structure stable. In western music, pitches related by a perfect fifth interval are nearest each other, and are most consonant when sound together. There are other rules: pitches a third apart are perceived to be more closely related than those a second apart; the major is regarded to be closer than the minor for both thirds and seconds; and the diminished fifth and augmented forth are more distant than other intervals. With these relationships of intervals, our measurement is built by considering distance between target channel and root of the current time chord, which is denoted by $A S_{N C}$.

To quantize those intervals between pitches, we follow
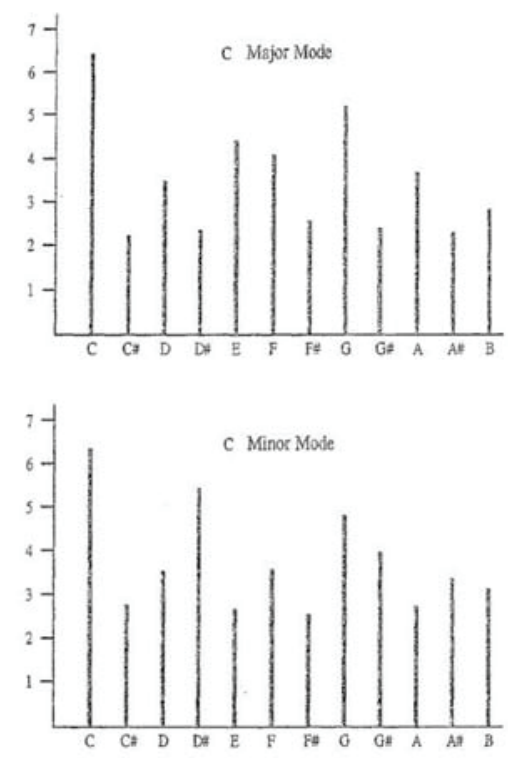

Fig. 2. Key profiles for $\mathrm{C}$ major and $\mathrm{C}$ minor, based on ratings for probe tones following the context (redrawn here).

Chew's idea, ${ }^{15}$ which models musical intervals as shown in Table 2.

In Chew's model, she divided basic intervals into 6 classes, intervals within the same class are treated equally. She also gave a constraint on the distances: $\frac{h}{r} \in$ $\left[\sqrt{\frac{2}{15}}, \sqrt{\frac{3}{15}}\right]$, where $r$ is the radius of the cylinder, while $h$ is the height gain per quarter rotation in the spiral array model.

$D_{C h}(i, j)$ denote Chew's distance between channel $i$ and $j$, and here we set $\frac{h}{r}=\sqrt{\frac{3}{15}}$, and $r=1$, then $A S_{N C}$ can be calculated as:

$$
\begin{aligned}
& A S_{N C}(t, i)=\left\{\begin{array}{cc}
\sqrt{2} / D_{C h}((i+10) \bmod 12, r(t)) & i \neq r(t) \\
1 & i=r(t)
\end{array}\right. \\
& i=1,2, \ldots, 61
\end{aligned}
$$


Here $r(t)$ is the root of chord at time $t$, which is detected by our proposed method in our previous study ${ }^{21}$.

So the musicological measurement of AS is shown here, which is a 2-dim feature, MAS:

$$
\text { Musicological } A S=\left(\begin{array}{l}
A S_{N K} \\
A S_{N C}
\end{array}\right)
$$

Finally, we use $A S$, a 5-dim feature vector (the first 3 dimensions are acoustical measurements from the AAS feature, while the latter 2 are musicological measurements from MAS feature), to detect salient pitches for each time stamp:

\section{Cognitive Melody Stream Transcription}

\subsection{Framework of our method}

As seen in Fig. 3, given a music piece, our method of melody stream transcription firstly relies on CQT, a "musical version" of STFT, ${ }^{20,22}$ which provides a more reasonable representation in frequency domain to describe the characteristic of harmonic structure of music sound by setting frequency components according to musical relations between tones. Then pitch tuning is implemented on the spectrum, this step is to reduce frequency deviation that results from difference between

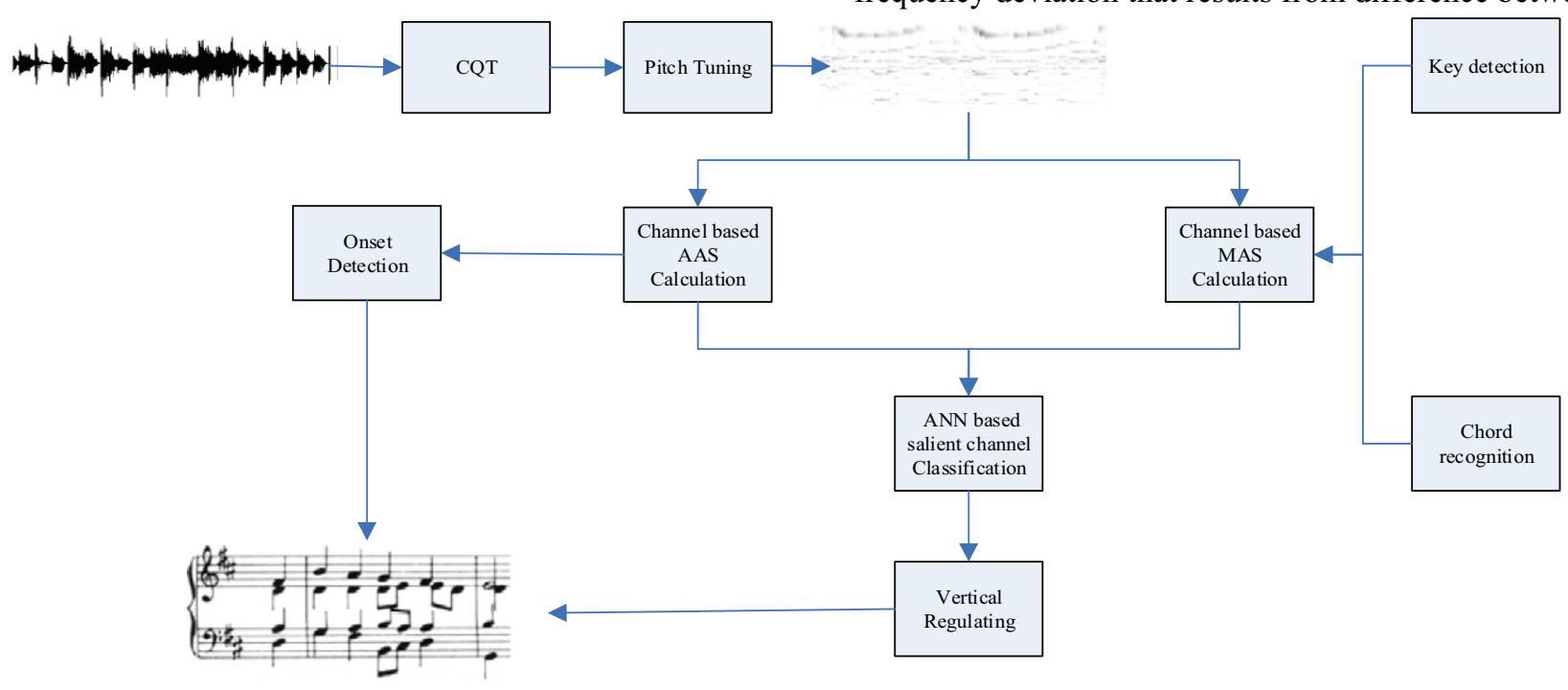

Fig. 3. Framework of our melody stream transcription method.

$$
A S=\left(\begin{array}{c}
A S_{L} \\
A S_{T-c h} \\
A S_{T-c r} \\
A S_{N K} \\
A S_{N C}
\end{array}\right)
$$

Table 2. Quantization of pitch intervals

\begin{tabular}{ll}
\hline Interval & Distance $^{2}$ \\
\hline Perfect fifth, perfect forth & $2 r^{2}+h^{2}$ \\
Major third, minor sixth & $16 h^{2}$ \\
Minor third, major sixth & $2 r^{2}+9 h^{2}$ \\
Major second, minor seventh & $4 r^{2}+4 h^{2}$ \\
Minor second, major seventh & $2 r^{2}+25 h^{2}$ \\
Diminished fifth, augmented & $4 r^{2}+36 h^{2}$ \\
forth & \\
\hline
\end{tabular}

actual pitch and standard numbering octave registers. ${ }^{21}$ Then we divide the spectrum into 61 channels with a band width of 100 cents, and center of each channel lies on the frequency of a chromatic tone. Then AAS of each channel is calculated by measuring the difference between distributions in several ways. The MAS is proposed to detect the salient structure of music sound mixture by using musicological knowledge and cognitive models, plus our previous work. ${ }^{20,21,23}$ Whereafter the AS (AAS+MAS) of all channels at each time are sent to a temporal ANN, and the salient channels are pick out by the network.

The temporal ANN is a 4-layer network with 2 hidden layers, which has a similar structure with Time Delay Neural Network (TDNN), and is capable of processing temporal sequence. Due to experiments, the network can process 61 feature vectors at one time to determine the salient channels. The input layer is fully interconnected 
to the first hidden layer, but each unit of the first hidden layer is just connected to 3 input units; the connection between the first and second hidden layer is similar, and each unit of the second layer looks at 5 units of the first hidden layer. There are 61 units in the output layer, according to 61 channels respectively, and each of them is connected to all of the second layer units. About the training method, it is a major contribution of our previous work $^{16}$. In that paper, we present the network training method called 'Entropy Error Function based training'. The method is based on EBP (Error Back-Propagation) training algorithm, but with ability of processing temporal sequence input and using a new training function called EEF (Entropy Error Function).

Another use of AAS is to detect onset of each tone. Finally, the chord sequence is made up with results of saliency detection after vertical regulating, and temporal information of onsets.

\subsection{Sequence timing-onset detection and post- processing}

As mentioned before, AAS is fairly sensitive to the beginning of a note. This is because AAS relies on "surprise" in the spectrum of the music signal: when a note sounds, the amplitude envelope increases quickly in attack stage, and an obvious change is observed. ${ }^{30}$ Thus, to capture temporal information of chord sequence, we apply AAS to detect onset (the beginning phase of the sound of a music note).

We use AAS as measurement of detecting onset: AAS is separately input to the temporal ANN described above, but the network is re-trained with only AAS information. Then the network detects saliency in each channel — if a channel is salient at a given time, then this time point is possibly an onset. The decision of onset follows 3 rules:

(i) There must be at least 3 salient channels at this time;

(ii) At least a 2-consecutive-octave interval that covers at least 2 salient channels can be found;

(iii) Salient channels that occur within $120 \mathrm{~ms}$ are treated as simultaneous saliency.

Polyphonic music consists of successions and superpositions of complex tones, and a key problem threats the transcription efficiency is false positive of salient pitch, which is led by harmonics of other complex tones. ${ }^{31}$ Hence, a post-processing method is still needed to improve precision of our detecting method. Here we propose a vertical regulating, which involves harmonic analysis of music signal and musicology.
If the fundamental of a complex tone is $f_{l}$, then its harmonics can be described by the following sequences:

$$
f_{1} ; 2 f_{1} ; 3 f_{1} ; 4 f_{1} ; \ldots . . n f_{1}
$$

When $n>8$, the harmonics are seldom audible, so we just consider harmonics limited to first eight. Here we do not consider the inharmonicity case which is caused by percussion instruments and high partials of string instruments in music. Here we treat the outputs of the ANN as note events sequence, and we deal with the problem on musicology level instead of frequency level. Thus we just follow the classic idea ${ }^{16}$ to regulate possible harmonic position and reduce false positive.

Then we rewrite the harmonic sequence denoted by frequency pattern, into a new form-the sequence consists of musical intervals that describe the distance between each harmonic and its fundamental:

0 oct; 1 oct; loct + P $5 ; 2$ oct 2 oct + M3; 2 oct + P $5 ; 2$ oct + m7; 3 oct (22)

Here, oct denotes octave, P5 is perfect fifth, M3 is major third, and $\mathrm{m} 7$ is minor seventh. For instance, the fifth harmonic of a given complex tone is 2 octaves plus a major third apart from its fundamental. So giving a salient pitch candidate, we can locate its harmonics based on interval sequence. If a channel is the harmonic of another (or itself), we say the former is "voted" by the latter.

So giving the detecting result set at a time, there must be some false alarms existing which are voted by more than one (besides itself) channel. Here we define salient pitch candidate set $C_{t}(m, n)$, and result set after regulating, $R_{t}(m, n)$ :

$C_{t}(m, n)$

$=\{i \mid m \leq i<n$, and $i$ is the salient pitch candidate $\}$

$m, n \in[1,61]$

$R_{t}(m, n)$

$=\{j \mid m \leq j<n$, and $j$ is the salient pitch after regulating $\}$

$m, n \in[1,61]$

Then vertical regulating is implemented as following.

Stage 1, for a time point $t$,

$$
\begin{aligned}
& R_{t}(1,61)=C_{t}(1,61) \\
& p=\min \left(C_{t}(1,61)\right) \\
& \% \% \text { Find } p \text { in } C_{t}(1,61), p \text { is the minimum } \\
& q=p+36 \\
& \text { while }(q<=61) \% \% \text { exit when } q=61 \\
& \text { for } i \text { in } C_{t}(p, q) \\
& \quad \text { if } i-j \text { meets }^{2}(22) \\
& \quad \operatorname{voter}_{i}=\text { voter }_{i}+1
\end{aligned}
$$


$\% \%$ For $i \in C_{t}(p, q)$, voter $_{i}$ is the number of channels $\% \%$ ( besides itself) which belong to $C_{t}(p, q)$, and at $\% \%$ the same time "vote" channel $i$, calculate voter ${ }_{i}$;

$$
\begin{gathered}
\text { if } \operatorname{voter}_{i}>2 \text { and } i-j>12 \\
\text { for } k=1: \text { length }\left(R_{t}(1,61)\right) \\
\text { if } R_{t}(1,61)(k)==\mathrm{i} \\
R_{t}(1,61)(k)=0
\end{gathered}
$$

$\% \%$ For $i \in C_{t}(p, q)$, if $\operatorname{voter}_{i}>2$, and the nearest $\% \%$ distance between $i$ and its voter (exclude itself)

$\% \%$ exceeds 1 octave, remove $i$ from $R_{t}(1,61)$;

$$
\begin{aligned}
& p=p+12 \\
& \text { if } p+36<61 \\
& \text { exit }
\end{aligned}
$$

$$
i=i+3
$$

Here we check the possibility that the salient candidates are false alarms in every 3 continuous octaves (36 semitones) by counting their voters. If a candidate has many distant voters, it tends to be a false alarm caused by its voters' harmonics. After stage 1, we get a salient harmonic structure at any given time point. Then on stage 2 , a smoothing process is implemented to ensure that these salient structures are "consecutive" in time, at least within time interval of the chord (note) they represent.

\section{Experiments}

In our experiments, a test set consists of single channel

Table 3. Salient pitch detection results (frame based).

\begin{tabular}{lllllll}
\hline & Classical & Pop & New age & Jazz & Folk & Total \\
\hline Frame & 57600 & 32400 & 57600 & 32400 & 18000 & 198000 \\
Salient pitch & 160150 & 89320 & 123410 & 60150 & 31560 & 464590 \\
Correct & 121719 & 45144 & 94178 & 42991 & 21433 & 325465 \\
False positive & 61974 & 51019 & 38241 & 26391 & 15314 & 192939 \\
False negative & 38431 & 44176 & 29232 & 17159 & 10127 & 139125 \\
Precision(\%) & 66.26 & 46.95 & 71.12 & 61.96 & 58.33 & 62.78 \\
Recall(\%) & 66.26 & 50.54 & 76.31 & 71.47 & 67.91 & 70.05 \\
F-measure & 0.71 & 0.49 & 0.74 & 0.66 & 0.63 & 0.66 \\
\hline
\end{tabular}

Stage 2, for a given music piece that can be divided into FrameNum frames,

$$
\begin{aligned}
& \text { While }(i<=\text { FrameNum }) \\
& \qquad \begin{aligned}
\text { for } j=i+1: i+2 \\
\text { for } k=1: 61 \\
\text { if } R_{i}(1,61)(k)=R_{j}(1,61)(k) \\
\qquad \operatorname{Comm}(i, j)=\operatorname{Comm}(i, j)+1
\end{aligned}
\end{aligned}
$$

$\% \%$ For each 3 -frame succession of salient structures, $\% \% \operatorname{Comm}(i, j)$ is the number of same channels between $\% \%$ salient structure $i$ and $j$,

$$
\text { if } \operatorname{Comm}(i, j)>1
$$$$
\text { if length(nonzeros } \left.\left(R_{i}(1,61)\right)\right)>
$$

length(nonzeros $\left.\left(R_{i}(1,61)\right)\right)$

$$
\begin{gathered}
R_{j}(1,61)=R_{i}(1,61) \\
\text { else } R_{i}(1,61)=R_{j}(1,61)
\end{gathered}
$$

$\% \%$ If exist $i, j$, make $\operatorname{Comm}(i, j)>1$, add the different $\% \%$ salient channels between $i$ and $j$ to each other and $\% \%$ make the salient structures of $i$ and $j$ equal execute stage 1 code $\% \%$ Re-do vertical regulating after smoothing wav files (44.1 kHz, 16 bit) is established. The set contains 16 classical music pieces (piano solo), 9 pop/rock (vocal, songs from Harte's Beattles collection $^{32}$ ), 9 jazz (piano solo), 16 new age (multiple instrument, songs from Secretgarden's famous album "Whitestones"), and 5 Chinese folk (vocal/instruments), all of which are 90s per segment. The test set contains real music and is diversified in genre and instrument selection. Also we have a training set for ANN, and the training set contains 200 20s MIDI (Musical Instrument Digital Interface) pieces and 220 20s real music pieces (all are different from the test set, the MIDI pieces are single pitch note sequences composed by ourselves, while real music pieces are from the same data source of test set). MIDIs are typical and representative music audio composed by us, such as single melodies formed by scales of major and minor keys, melody streams formed by typical triads. We build the MIDI set to enable the network to classify the fundamental and typical cases of real music through training. Then the real music segments are used to strengthen network's ability of 
generalization. The ground truth set of test data comes from published music scores (most are collected from music publications, others are online resource), or author's contribution (Harte's work ${ }^{32}$ ), with temporal information labeled by us. All the transcriptions are revised by music school students.
According to table 4, when $C L=50 \%$, our method performs a $71.05 \%$ recall level. For performance of each genre, pop and folk are still below average, because there are many false positives and negatives that have weakened consistency of salient frames within note. Furthermore, if we enhance the $C L$ requirement, the

Table 4. Salient pitch detection results (note based, $C L=50 \%$ ).

\begin{tabular}{lllllll}
\hline & Classical & Pop & New age & Jazz & Folk & Total \\
\hline Salient pitch & 7714 & 7022 & 7387 & 3750 & 1976 & 27849 \\
Correct & 5691 & 4468 & 5904 & 2543 & 1181 & 19787 \\
False negative & 2023 & 2554 & 1483 & 1207 & 795 & 8062 \\
Recall(\%) & 73.77 & 63.63 & 79.92 & 67.81 & 59.77 & 71.05 \\
\hline
\end{tabular}

Firstly, efficiency of onset detection is checked. For our onset detection results compared to ground truth ones, tolerance of time precision is from $+/-50 \mathrm{~ms}$ to less, which means given a ground-truth onset, if there is a detection in tolerance time-window around it, it is considered as correct. Here our AAS based method gets an equal precision, recall and F-measure of 0.79 . Compared to onset detection results in MIREX2011 $1^{33}$ (highest precision $83.0 \%$, highest recall $86.8 \%$, and highest F-measure 0.83), our AAS based onset detection is acceptable. The worse performances come from pops and folks: pop is fierce and vocal with drum noise, while Chinese folk is so strongly vocal that some of the onsets appear in the music score are not distinct in the continuous singing voice.

Table 3 are detection results of salient channels (frame by frame) by proposed method. Note that the salient pitch here may contain many repetitions, since a note lasts for many frames.

Here proposed framework gets a total performance of 0.66 in F-measure at frame level. For each genre, the result of new age is still stable, while that of pop is below $50 \%$; the method makes many false positives in jazz and folk, because music of the 2 genres is simple instrumented, but strong vocal.

Then our method is evaluated at note level in Table 4: for note based saliency detection, we define a variable of confidence level, $C L$, which denotes the required percentage of salient frames to confirm a channel is salient at note level. If a channel remains salient in more than $C L$ of the frames within current note, we regard it as a salient one at this time. problem of frame detection consistency aggravates: when $C L$ rise from $60 \%$ to $80 \%$, recall level of proposed method falls from 0.62 to 0.29 .

To examine the effectiveness of proposed musicological processes, we repeat the frame based experiment with a missing post method (post-processing is removed from our system - outputs of network are the final results), a missing MAS method (only AAS feature is used to detect saliency in channels), and a perfect MAS method (keys and chord roots are man-labeled instead of results in our previous work ${ }^{15}, 16$, which can be treated as perfect information instead of deficient ones).

Table 5. Results comparison of MAS feature and postprocessing method

\begin{tabular}{llll}
\multicolumn{3}{c}{ postprocessing method } \\
\hline & $\begin{array}{l}\text { Missing } \\
\text { post }\end{array}$ & $\begin{array}{l}\text { Missing } \\
\text { MAS }\end{array}$ & $\begin{array}{l}\text { Perfect } \\
\text { MAS }\end{array}$ \\
\hline Frame & 198000 & 198000 & 198000 \\
Salient pitch & 464590 & 464590 & 464590 \\
Correct & 293860 & 309895 & 360577 \\
False positive & 304735 & 212784 & 124671 \\
False negative & 170730 & 154695 & 104013 \\
Precision(\%) & 49.09 & 59.29 & 74.31 \\
Recall(\%) & 63.25 & 66.70 & 77.61 \\
F-measure & 0.55 & 0.63 & 0.76 \\
\hline
\end{tabular}

According to the experiments in Table 3 and Table 5, adding post-processing into our system improves the recall, and it also drives the precision $13.6 \%$ higher. For the importance of MAS, it seems the performance doesn't fall much when MAS is missing, but when changing to precise key and chord root information (from the music score) based MAS, detection results are 
inspiring. Hence, key point for an effective MAS is the accuracy of its background information.

Furthermore, we compare the ability of harmonic structure detection of our method with Daniel Ellis' chord detection system submitted to MIREX 2010.34 Ellis' system reached an accuracy of $74.96 \%$ in the competition, and here we adopt their EW1 method. Due to the detection of augmented/diminished chords are not in EW1's consideration, we re-train the model to get a EW12, which is able to detect augmented/diminished chords. Here accuracy of chord detection is the
Each synthetic music piece is segmented into smaller units in different time scales, paired with its source music. Then 2 groups of participants ( 3 people in each group, without music background) are randomly given the small segments pair by pair, and asked to rate similarity between the synthetic segment and the source one with 0 (not similar), 1 (somewhat similar), 2 (very similar). Then test data are exchanged between groups. So each pair of test segment is evaluated twice. For those segments that get different scores in the 2 rounds, 3 participants are asked to vote for deciding the final score.

Table 6. Comparison of salient harmonic structure detection with Ellis' method.

\begin{tabular}{lccc}
\hline & & & \\
& Accuracy of EW1(\%) & Accuracy of EW12(\%) & Accuracy of our method(\%) \\
\hline Classical & 68.48 & 66.82 & 68.58 \\
New age & 69.01 & 68.17 & 72.15 \\
Jazz & 62.85 & 60.79 & 67.74 \\
Pop & 60.45 & 57.36 & 62.07 \\
Folk & 56.47 & 53.88 & 60.32 \\
Total & 65.17 & 63.48 & 67.64 \\
\hline
\end{tabular}

percentage of total time of correct harmonic structure. To compare our method with Ellis' system, when we mention "correct" harmonic structure at some time point, we mean the triad structure can be detected perfectly, other salient components are ignored. From Table 6 we can see, for typical triad structure detection, our method overperforms the EW1 and EW12 system.

Obviously, our method has reached an acceptable performance through above objective evaluations. To prove this efficiency, we design experiments to test our cognitive transcription method through human's perception. Firstly, musical software Overture is used to compose electronic music according to our transcription.
The simple rating method introduced is to reduce confusion of similarity level caused by complex score rule. Rating results of $3 \mathrm{~s} /$ segment, are shown in Table 7 . In addition to the $3 \mathrm{~s} /$ segment evaluation, we also rate $4 \mathrm{~s} /$ segment and $6 \mathrm{~s} /$ segment(average score 1.31 and 1.33 respectively): as length of segment increases, average score and total percentage of more similar segments are augmented, which both indicates similarity between synthetic segments and source ones increase. Furthermore, for each type of segment length, average score is above 1.29 , which is a higher similarity level with respect to the basic reference level score of 1 . Meanwhile, around $90 \%$ of synthetic segments are considered to be similar with source ones, which is

Table 7. Result of 3s/segment subjective evaluation.

\begin{tabular}{lllllll}
\hline & Classical & Pop & New age & Jazz & Folk & Total \\
Total segment & 480 & 270 & 480 & 270 & 150 & 1650 \\
0 point segment & 43 & 44 & 30 & 17 & 21 & 155 \\
1 point segment & 211 & 149 & 223 & 181 & 98 & 862 \\
2 point segment & 226 & 77 & 227 & 72 & 31 & 633 \\
Average score & 1.38 & 1.12 & 1.41 & 1.20 & 1.07 & 1.29 \\
Percentage of 1 \& 2 point segment(\%) & 91.04 & 83.70 & 93.75 & 93.70 & 86.00 & 90.60 \\
\hline
\end{tabular}


inspiring. For each genre, pops and folks are below average, but average scores of both are above 1 . Contrasting with precision and recall evaluation before, performance of subjective similarity is better, which consists with theory about self learning and selective attention of human's brain, and fact that structure of chord is so flexible that in many cases adding notes into it won't change the way it sounds (but aesthetic feeling will be spoiled by inconsonance).

\section{Conclusions}

\subsection{Conclusions}

In this paper we've presented an approach for melody stream transcription. In particular, we have addressed the issue of salient structure detection in polyphonic music with a cognitive feature, AS. AS takes both acoustical attention selectivity and tonal context dependence into consideration, so it is effective to detect salient channels which are composed of the chord structure at every time point. To make our method robust, we also propose vertical/ horizontal algorithm for regulating melody stream.

Due to experiments, our salient pitch method reaches a precision of $62.78 \%$, and a recall of $70.05 \%$ at frame level, and post processing algorithm also works well. Furthermore, perfect tonal information (key, chord) can improve performance of saliency detection most, indicating needs for reliable method of key/chord recognition. The propose method performs well in subjective evaluation, the average score is 1.29 , and around $90 \%$ of synthetic segments are considered to be similar with their original input melody.

\subsection{Future work}

In the next stage of our research, since there are still many unclear aspects on human's perception of music, we plan to enrich our auditory saliency theory by doing some fundamental research in cognition. Firstly, we will study musicology related cognitive problem through neuroscience science method using Event Related Potential (ERP) and functional Magnetic Resonance Imaging (fMRI) techniques. We plan to design task related experiment to investigate into human's perception of tonal elements (key, chord, melody). In this way, we might be able to clarify how musicology affects music perception contextually and structurally, so that we can improve the MAS feature.
Secondly, we will investigate into auditory selective attention theory to figure out if there exists a more reasonable representation of auditory saliency spatiotemporally, so that the AAS feature can be measured more elaborately. This will be done also with the help of psychological experiments.

Thirdly, with respect to the application fields of this method, we plan to build a prototype system for music transcription by using our framework, in order to enhance the performance of the method, and make it more comparable to others methods. About the data corpus, a bigger test set will be built and music of more genres and instruments will be selected. Furthermore, more testing data from other platforms will be imported into our test set to make sure that our framework will be more open.

\section{Acknowledgements}

This work is supported by the National Natural Science Foundation of China, under the grant of No.61171186, Research Funds for Technological Innovation Talents of Harbin City (Grant No. 2013RFQXJ112), and Fundamental Research Funds for the Central Universities (Grant No. HIT.NSRIF.2013097).

\section{References}

1. A. Cemgil, Bayesian Music Transcription (Ph.D. dissertation, Radboud University Nijmegen, 2004)

2. M. A. Casey, R. Veltkamp, M. Goto, M. Leman, C. Rhodes and M. Slaney, Content-Based Music Information Retrieval: Current Directions and Future Challenges, Proceedings of IEEE. 96(4) (2008) 668-696.

3. Y. Zhu and M. Kankanhalli, Precise pitch profile feature extraction from musical audio for key detection, IEEE Transactions on Multimedia. 8(3) (2006) 575-584.

4. E. Gomez, P. Herrera, Estimating the tonality of polyphonic audio files: cognitive versus machine learning modeling strategies, Proc. ISMIR (Barcelona, Spain, 2004).

5. M. Goto, A predominant-F0 estimation method for $\mathrm{CD}$ recordings: MAP estimation using EM algorithm for adaptive tone models, Proc. ICASSP 2001 (2001), pp. 3365-3368.

6. A. Klapuri, Multipitch analysis of polyphonic music and speech signals using an auditory model, IEEE Trans. Audio, Speech and Language Processing. 16(2) (2008) 255-266.

7. V. Emiya, R. Badeau and B. David, Multipitch estimation of piano sounds using a new probabilistic spectral smoothness principle, IEEE Trans. Audio, Speech and Language Processing. 18(6) (2010) 1643-1654. 
8. K. Lee and M. Slaney, Acoustic chord transcription and key extraction from audio using key-dependent HMMs trained on synthesized audio, IEEE Trans. Acoust., Speech Language Process. 16(2) (2008) 291-301.

9. S. Jo, S. Joo and C. D. Yoo, Melody pitch estimation based on range estimation and candidate extraction using harmonic structure model, in Interspeech, pp. 2902-2905, (Makuhari, Japan, 2010).

10. T.-C. Yeh, M.-J. Wu, J.-S. Jang, W.-L. Chang and I.-B. Liao, A hybrid approach to singing pitch extraction based on trend estimation and hidden Markov models, in IEEE Int. Conf. on Acoustics, Speech and Signal Processing (ICASSP) (Kyoto, Japan, 2012), pp. 457-460.

11. V. Arora and L. Behera, On-line melody extraction from polyphonic audio using harmonic cluster tracking, IEEE Trans. on Audio Speech and Language Processing. 21(3) (2013) 520-530.

12. J. Salamon and E. Gomez, Melody extraction from polyphonic music signals using pitch contour characteristics, IEEE Trans. on Audio Speech and Language Processing. 20(6) (2012) 1759-1770.

13. J. Salamon, E. Gomez, D. P. W. Ellis and G. Richard, Melody extraction from polyphonic music signals: approaches, applications and challenges, IEEE Signal Processing Magazine. 31(2) (2014) 118-134.

14. C. Krumhansl, Cognitive foundations of musical pitch (Oxford University Press, New York, 1990).

15. E. Chew, Towards a mathematical model of Tonality (Ph.D. dissertation, MIT, 2000).

16. E. Terhardt, G. Stoll and M. Seewann, Algorithm for extraction of pitch and pitch salience from complex tonal signals, Journal of the Acoustical Society of America. 7 (1982) 679-688.

17. D. Temperley, Music and probability (MIT Press, Cambridge, MA, 2007).

18. C. Krumhansl, The cognition of tonality - as we know it today, J. New Music Research. 33 (2004) 253-268.

19. D. Deutsch, The Psychology of Music, 2nd ed. (Academic, San Diego, CA, 1999).

20. J. Sun, H. Li and L. Lei, Key detection through pitch class distribution model and ANN, Proc. DSP2009, (Santorini, Greece, 2009).

21. J. Sun, H. Li and L. Lei, ANN Based Music Chord Perception, J. Tsinghua University (Sci \& Tech). 49(S1) (2009) 1369-1374, 1379.

22. J. Brown, Calculation of a constant $\mathrm{Q}$ spectral transform, J. Acoust. Soc. Amer. 92(1) (1991) 425-434.

23. J. Sun, H. Wang and J. Liu, A cognitive method for melody stream transcription, CCIS2014, (Hong Kong, 2014).

24. L. Itti and C. Koch, Computational modeling of visual attention, Nature Reviews, Neuroscience. 2 (2001) 194203.

25. N. Bruce and J. Tsotsos, Saliency, attention, and visual search: An information theoretic approach, Journal of Vision. 9(3) (2009) 1-24.
26. L. Itti and P. Baldi, Bayesian Surprise Attracts Human Attention, Advances in Neural Information Processing Systems. 18 (2006) 547-554.

27. E. Jaynes, Probability Theory: The Logic of Science (Cambridge University Press, 2003).

28. S. Kullback, The Kullback-Leibler distance, The American Statistician. 41 (1987) 340-341.

29. B. Hyer, The New Grove Dictionary of Music and Musician, S. Sadie, edited. (Macmillan, New York, 2001).

30. J. Bello, C. Duxbury, M. Davies and M. Sandler, On the use of phase and energy for musical onset detection in the complex domain, IEEE Signal Processing Letters. 11(6) (2004) 553-556.

31. A. Bregman, Auditory scene analysis: the perceptual organization of sound (MIT Press, 1990)

32. C. Harte, M. Sandler, S. Abdallah and E. Gomez, Symbolic representation of musical chords: A proposed syntax for text annotations, Proc. Int. Conf. Music Information Retrieval, (London, U.K., 2005), pp. 66-71.

33. Onset detection results page of MIREX 2011: http://nema.lis.illinois.edu/nema_out/mirex2011/results/a od/summary.html

34. Chord detection results page of MIREX 2010: $\mathrm{http} / / /$ nema.lis.illinois.edu/nema_out/mirex2010/results/a ce/ 